\title{
GROSSESSE APRES FECONDATION IN VITRO DANS UN CAS D'EJACULATION SECHE (ABSENCE D'EMISSION) PARTIELLEMENT AMELIORE PAR TRAITEMENT PHARMACOLOGIQUE
}

\author{
J. Buvat, G. Marcolin, M. Buvat-Herbaut, O. Renouard, G. Couplet, Jean-Luc Dehaene
}

\author{
Association pour l'Etude de la Pathologie de l'Appareil Reproducteur et de la Psychosomatique
} EPARP, 47-49 rue de la Bassée, 59000 LILLE (FRANCE)

\begin{abstract}
PREGNANCY FOLLOWING IN VITRO FERTILIZATION IN A CASE OF EMISSION FAILURE ONLY PARTLY IMPROVED BY PHARMACOLOGICAL TREATMENT. To date only 3 pregnancies have been reported following treatment of emission failure. We report the fourth one obtained from a male with an history of retroperitoneal lymphadenectomy. Pharmacological treatment induced antegrade ejaculation of a small semen drop wich was unsuccessfully inseminated during 15 cycles. In Vitro Fertilization (IVF) obtained a good fertilization rate and full-term twin pregnancy at the third attempt. IVF clearly widens the possibility of treating infertility in case of failure of ejaculation. Keyswords : emission failure, pharmacological treatment, In Vitro Fertilization. Andrologie, 1991, $1: 69-70$.
\end{abstract}

L'éjaculation normale requiert : 1. l'émission du sperme dans l'urètre postérieur ; 2 . la fermeture du sphincter interne de la vessie ; 3. l'expulsion du sperme par des contractions musculaires. L'absence d'éjaculation externe peut provenir de 3 mécanismes différents : l'éjaculation sèche, ou absence d'émission est due à l'échec de la première phase ; l'éjaculation rétrograde est due à celui de la seconde ; l'anéjaculation sans orgasme, ou éjaculation retardée, est due à celui de la troisième (3). Contrairement aux deux dernières perturbations, très peu de grossesses ont été obtenues en cas d'éjaculation sèche, qui résulte généralement d'anomalies neurologiques. Nous rapportons ici le tème cas connu, obtenu en associant un traitement pharmacologique à la Fécondation In Vitro (FIV).

\section{CAS CLINIQUE}

Un homme de 36 ans consulta dans nolve centre pour infécondité primaire. Il avait des antécédents de séminome traité 7 ans plus tôt par orchidectomie droite, curage ganglionnaire rétropéritonéal, et bléomycine pendant 2 mois. Son éjaculation disparut après le curage. Le diagnostic d'éjaculation sèche fut basé sur la persistance de l'orgasme, excluant l'anéjaculation sans orgas- me, et l'absence de spermatozoides dans les urines émises après orgasme à 3 reprises différentes, excluant l'éjaculation rétrograde. Le reste du bilan ne révéla pas d'autre facteur d'infertilité : le testitule restant avait subi l'hypertrophie compensatrice physiologique, les taux sériques de FSH et de testostérone étaient normaux, le bilan de la partenaire l'était également.

Un traitement pharmacologique fut d'abord essayé, sous la forme de RUPTON ${ }^{R}$ (Laboratoire DEXO, Nanterre, France), qui associe un sympathomimétique (chlorhydrate de norephedrine, 50 $\mathrm{mg} /$ comprimé) et un parasympathicolytique (maleate de brompheniramine, $4 \mathrm{mg} / \mathrm{cp}$ ). La dose maxima tolérée (3 à $4 \mathrm{cps} / \mathrm{j}$ ) induisit une éjaculation antégrade de très faible volume $(0.2$ à 0.5 $\mathrm{ml}$ ), contenant 35 à 144 millions de spermatozoïdes/ml. Ceux-ci avaient une morphologie normale (tératospermie 17 à $46 \%$ ) et une mobilité céduite (mobilitế normalement prospective à 1 $h: 15$ à $25 \%$ ). Cependant la survie des spermatozoïdes s'avéra normale dans certains milieux comme la glaire cervicale (mobilité $70 \%$ à 4 h) et le milieu de Menezo B2 (mobilité $60 \%$ à 24 h). Il ne fut pas trouvé de spermatozoïdes supplémentaires dans les urines.

Le test post-coital, vérifié à 3 reprises, restant négatif, l'éjaculat antégrade obtenu sous RUP$\mathrm{TON}^{\mathrm{R}}$ fut utilisé pour inséminations. Dix cycles d'inséminations cervicales, faites à domicile par le mari, puis 5 cycles d'inséminations intra-utérines synchronisées avec l'ovulation, faites avec des spermatozoïdes lavés et resuspendus dans le Menezo B2 après stimulation ovarienne par des ménotrophines (hMG) et de l'hormone gonadotrope chorionique (hCG) sous monitorage échographique et hormonal étroit échouèrent. A ce stade, la femme avait 36 ans, et le couple accepta de tenter une FIV, traitement qui fut répété à 2 reprises du fait de la survenue d'une fécondation lors du premier essai.

La stimulation ovarienne utilisà le citrate de clomifène et des hMG lors du ler essai, puis une association du LHRH-agoniste DRTP 6 - LHRH (DECAPEPTYL ${ }^{R}$, Laboratoire IPSEN/BIOTE$\mathrm{CH}$, Paris) et d'hMG. L'épouse s'averra être une répondeuse faible, ce qui fait que les 3 ponctions transvaginales échoguidées n'obtinrent respecti- vement que 1,1 et 3 ovocytes. Les spermatozoïdes furent préparés par migration ascendante, qui obtint des concentrations de respectivement $1.2,0.85$ et 1.4 Millions $/ \mathrm{ml}$, avec des survies à 24 h de $10 \%, 30 \%$ et $40 \%$. Un, un et deux embryons furent obtenus et transférés. La femme conçut après le 3ème transfert, et accoucha de jumeaux ( 1 fille et 1 garçon) en Juillet 1988, au terme d'une grossesse sans problème.

\section{DISCUSSION}

Schill et Bollman (5) ont rapporté la première grossesse obtenue dans un cas d'éjaculation sèche due à un curage ganglionnaire rétropéritonéal. Ils avaient inséminé des spermatozoïdes récupérés dans les urines après qu'un agent sympathomimétique, le Midodrin, ait induit une éjaculation rétrograde. Le second cas a également été rapporté par Schill (4). Il concernait un homme de 35 ans, atteint de diabète juvénile, et présentant une éjaculation sèche incomplète qui fut traitée avec succès par la brompheniramine pendant 2 ans. Cet agent parasympathicolytique induisit une éjaculation antégrade normale, et finalement la femme conçut après rapport sexuel normal. Récemment Blank et al (2) ont rapporté une 3ème grossesse obtenue par électroéjaculation suivie d'un Zygote Intra-Fallopian Transfer (ZIFT) après échec des inséminations dans un cas d'éjaculation sèche consécutif à une plaie abdominale par balle.

Les procréations assistées ont également obtenu des succès dans d'autres types de perturbations organiques de l'éjaculation, après échec des inséminations synchronisées avec l'ovulation : 2 grossesses après Gamete-Intra-FallopianTransfer (GIFT) dans des cas d'ejaculation rétrograde (6), 1 grossesse après électro-éjaculation puis FIV chez un paraplégique (1). FIV, GIFT et ZIFT élargissent manifestement considérablement les possibilités de traitement de la stérilité par anéjaculation, du fait qu'elles ne nécessitent qu'un nombre relativement modéré de spermatozoïdes. Elles devraient être essayées non seulement lorsque les inséminations échouent, mais également d'emblée dans les cas où traitement pharmacologique, vibrateur, électro-éjaculation, récupération des spermatozoïdes dans les usines et/ou prélèvement chirurgical de spermatozoïdes 
déférentiels obtiennent des spermatozoïdes mobiles, mais en nombre trop faible (moins de 0.5 à 1 million) pour donner une chance réelle de succès après insémination intra-utérine.

REMERCIEMENTS : à Mlle Laurence FREALLE pour la préparation de ce manuscrit.

\section{REFERENCES}

1- Ayers JWT, Moinipanah R, Bennett CJ, Randolph JF, Peterson EP : Successful combination therapy with electroejaculation and in vitro fertilizationembryo transfer in the treatment of a paraplegic male with severe oligoasthenospermia. Fertil. Steril. 1988, 49 : 1089-1090.

2 - Blank W, Batzofin J, Tan T, Hubert G, Serafini P : The use of electroejaculation after a major gunshot wound to the abdomen : a unique application. Fertil. Steril., 1990, 54 : 950-952.
3- Buvat J : Anéjaculation et éjaculation asthéniques : abord clinique et thérapeutique, In "L'éjaculation et ses pertubations", J. Buvat et P. Jouannet Eds, SIMEP, Villeurbanne, France, 1984, 35-48.

4 - Schill WB : Successful treatment of emission failure in a diabetic using brompheniramine : A case report. Proceedings of the Vth International Congress of Andrology, Florence, 1989, p 212.

5 - Schill WB, Bollmann W: Aspermia due to retroperitoneal lymphadenectomy : first report of pregnancy after insemination of spermatozoa obtained by midrodrin-induced retrograde ejaculation. Proceedings of the XI World Congress on Fertility and Sterility. Dublin, 1983, Abstract 260.

6 - Vernon M, Wilson E, Muse K, Estes S ; Curry S. Successful pregnancies from men with retrograde ejaculation with the use of washed sperm and gamete intrafallopian tube transfer (GIFT). Fertil. Steril. 1988, $50: 822-824$.
RESUME : Il n'a été rapporté jusqu'ici que 3 grossesses après traitement de stérilités par éjaculation sèche. Nous rapportons la 4ème, obtenue à partir d'un homme avec antécédents de curage ganglionnaire rétropéritonéal. Le traitement pharmacologique induisit l'éjaculation antégrade d'une petite goutte de sperme dont l'insémination pendant 15 cycles n'obtint pas de grossesse. La Fécondation In Vitro (FIV) obtint un bon taux de fécondation, et une grossesse gémellaire menée à terme au Jème essai. La FIV élargit manifestement les possibilités de traitement des infertilités par anéjaculation. Mots clés : éjaculation sèche, absence d'émission, traitement pharmacologique, Fécondation In Vitro. Andrologie, 1991, 1 : 69-70. 